Enfoques. Revista de Investigación en Ciencias de la Administración http://doi.org/10.33996/revistaenfoques.v4i13.79 No. 13 | Volumen 4 | Enero - Marzo 2020

\section{DIDÁCTICA DE LOS DOCENTES DEL PROGRAMA DE TECNOLOGÍA TEXTIL DEL SENA MEDELLÍN, COLOMBIA}

\author{
DIDACTICS OF TEACHERS FROM THE TEXTILE TECHNLOGIES \\ PROGRAM, OFFERED BY SENA MEDELLIN, COLOMBIA
}

Claudia Patricia Gómez Peláez y Luis Fernando Melchior Ramírez

Artículo recibido octubre 2019 | Arbitrado noviembre 2019 | Publicado 01 de enero 2020

\section{Resumen}

El objetivo de esta investigación fue caracterizar la didáctica utilizada por los docentes de la oferta cerrada del programa de Tecnología Textil impartido en el Centro Textil y de Gestión Industrial del Sena, Medellín, en las cohortes de tercero, quinto, séptimo y octavo trimestres en formación para la vigencia 2018. Esta investigación fue descriptiva, se desarrolló desde la comprensión holística de la ciencia y utilizó el método holopráxico. El diseño fue de campo univariable, contemporáneo y transeccional. Para medir didáctica docente, se utilizó la técnica de encuesta, y el instrumento fue un Cuestionario de Didáctica docente conuna confiabilidad de 0,80. Estos aprendices se encontraban cursando tercero, quinto, séptimo y octavo trimestre. Para el análisis se utilizó estadística descriptiva, y se encontró que la didáctica utilizada por los instructores de las tres asignaturas consideradas, se ubicó dentro de las categorías buena y muy buena, y el comportamiento de los docentes fue bastante homogéneo en este evento.

Palabras clave: Educación, didáctica; formación profesional; tecnología textil

\section{Abstract}

The objective of this research was to characterize the didactic used by teachers of the closed offer of the Textile Technology program taught at the Textile and Industrial Management Center of the Seine, Medellín, in the cohorts of third, fifth, seventh and eighth quarters in training for the validity 2018. This research was descriptive, was developed from the holistic understanding of science and used the holopraxic method. The design was univariable, contemporary and transectional. To measure teaching didactics, the survey technique was used, and the instrument was a Teaching Didactics Questionnaire with a reliability of 0.80 . These apprentices were in third, fifth, seventh and eighth trimesters. For the analysis, descriptive statistics were used, and it was found that the didactics used by the instructors of the three subjects considered, were within the good and very good categories, and the behavior of the teachers was quite homogeneous in this event.

Key words: Education, didactics, teaching, professional education, textile technologies
Claudia Patricia Gómez Peláez cpatriciagp@yahoo.com.ar Sena Medellín, Colombia

Técnico Textil con Especialización en Control de Calidad, Tecnólogo Textil, Especialización Tecnológica en Gestión del Talento Humano, Administrador de Empresas, Magister en Administración y Planificación Educativa.

Luis Fernando Melchior Ramírez luisfernandomelchiorr@yahoo.com Pontificia Universidad Javeriana de Bogotá, Colombia.

Ingeniero Electrónico, Magister en Administración y Planificación Educativa. Trabaja actualmente en la Pontificia Universidad Javeriana de Bogotá, Colombia. 


\section{INTRODUCCIÓN}

A lo largo de la historia la educación ha jugado un papel fundamental en el surgimiento de grandes civilizaciones $y$ múltiples avances tecnológicos, culturales, sociales, en la salud y en la economía. Existe una relación mutua entre la evolución del ser humano y la educación, pues una ha permitido y motivado a la otra, y sin educación no se puede hablar de evolución, así como tampoco sin evolución se puede hablar de avances en educación.

De esta forma, la educación se convierte entonces en un motor fundamental para impulsar a cada país hacia el desarrollo, para lo cual es indispensable que las personas pasen por procesos educativos de calidad que los convierta en sujetos orientadores de la transformación, que a su vez favorezcan oportunidades de progreso y desarrollen valores éticos que permitan formar mejores seres humanos.

El desarrollo tecnológico actual lleva a formar trabajadores del conocimiento que puedan afrontar los retos que implica el paso de la era industrial a la era de la información, con los correspondientes requisitos en cuanto a recursos humanos postindustriales y para un mundo del trabajo en plena evolución (Unesco-Unevoc, 2010).

Para el caso de Colombia, dentro de la misión del Servicio Nacional de Aprendizaje (SENA), se encuentra el cumplir la función que le corresponde al Estado de invertir en el desarrollo social y técnico de los trabajadores colombianos, al ofrecer $y$ ejecutar la formación profesional integral mencionada en la Ley 119 para la incorporación y el desarrollo de las personas en actividades productivas que contribuyan al desarrollo social, económico y tecnológico del país (Sena, 2017).

De esta manera el Sena, a través del Centro Textil y de Gestión Industrial de la
Regional Antioquia y del Centro de Manufactura en Textil y Cuero de la Regional Distrito Capital, ofrece el programa de formación profesional integral en Tecnología Textil, el cual, con sus egresados, fortalece uno de los sectores económicos más importantes del país, porque, tal como lo menciona Jara (2000), la industria textil colombiana ha sido determinante en el desarrollo industrial del país, al construir una cadena productiva muy compleja y diversificada.

Para Conde (2009) una de las industrias clave de la nación es el sector textilconfección ya que aporta el $9 \%$ del Producto Interno Bruto del país, $24 \%$ del empleo en manufactura y $7 \%$ del total de las exportaciones. Según González (2018), el sector textil y el de la confección eran claves para el crecimiento económico de Antioquia para 2018, pues dichos renglones aportaban el $2,5 \%$ del PIB de Antioquia, y contribuían con el $29 \%$ del valor agregado del total de las industrias de la ciudad de Medellín.

En consecuencia, es responsabilidad del Sena velar por el buen rendimiento académico y por la formación profesional de calidad de sus aprendices. Esta formación profesional requiere de docentes capaces de desarrollar una didáctica acorde con las competencias que le van a permitir a los aprendices enfrentar los retos del mercado laboral. Por ello resulta de sumo interés describir la didáctica utilizada por los docentes en la formación de estos aprendices.

Según la Organización Internacional del Trabajo (OIT) la formación profesional en América Latina y el Caribe es un campo estratégico para lograr avances en la promoción del trabajo decente, donde el tema de las políticas públicas de formación para el trabajo ha vuelto al centro de la agenda de los ministerios de trabajo en la 
política de los gobiernos. Todo esto se debe a la acelerada transformación en la que se encuentra el mundo del trabajo, y a la necesidad de aprovechar las oportunidades generadas por la integración de la economía mundial, donde la calificación y la formación profesional se han puesto como un desafío esencial para avanzar en el desarrollo económico y social de los países.

La formación profesional suele asociarse comúnmente a la educación universitaria, sin embargo, no todo profesional ha obtenido su título a través de una universidad, sino a través de otras instituciones de educación superior. Por otra parte, en la actualidad el término educación superior ha sido reemplazado por el término educación terciaria, el cual es un término de un alcance más amplio y universal.

Tanto la Eftp como la educación universitaria son los dos pilares de la educación terciaria. La educación terciaria se concibe como una organización de los diferentes niveles de educación post-media, y se vincula con las necesidades sociales y las del mercado laboral al conectarse con las necesidades de los diferentes sectores de la economía. Así mismo, la Educación Terciaria cuenta con los mecanismos para diferenciar sus dos pilares en varios aspectos, dentro de los cuales se destacan sus características, objetivos, vocación, niveles de especialización, ocupaciones hacia las que se dirigen y el aseguramiento de la calidad. También, cuenta con las herramientas para la movilidad académica y laboral a través del reconocimiento de los aprendizajes a lo largo de la vida independientemente de cómo hayan sido adquiridos (Ministerio de Educación Nacional de Colombia, 2015).

Para el caso de Colombia, dentro de la misión del Servicio Nacional de Aprendizaje (SENA) se encuentra el cumplir la función que le corresponde al Estado de invertir en el desarrollo social y técnico de los trabajadores colombianos, al ofrecer $y$ ejecutar la formación profesional integral mencionada en la Ley 119, para la incorporación y el desarrollo de las personas en actividades productivas que contribuyan al desarrollo social, económico y tecnológico del país (Sena, 2017).

El Sena nace como un establecimiento público de orden nacional en 1957 como iniciativa entre los trabajadores, los empresarios, la iglesia católica y la Organización Internacional del Trabajo, así mismo se encuentra adscrito al Ministerio de Trabajo y Seguridad Social de la República de Colombia; ofrece formación gratuita teóricopráctica para que las personas adquieran y mejoren destrezas, aptitudes, valores y conocimientos técnicos y tecnológicos que les permitan desarrollar sus capacidades para actuar crítica y creativamente en la actividad productiva, para de esta forma contribuir a su propio desarrollo personal, a la competitividad de la economía y al ejercicio pleno de la ciudadanía (Sena, 2017).

El comportamiento del sector textil y confección comparte la mayoría de las dificultades de la industria manufacturera nacional, tales como la competencia nacional e internacional legal e ilegal, fluctuaciones de la moneda, productividad y costos de materia prima. Desde el año 2000 al 2010 se evidencia un crecimiento en el sector, pero en el 2009 sufrió una crisis de la cual se empieza a recuperar en los años 2011 y 2012. Para el año 2014 se muestra un decrecimiento del sector textil en $-3,5 \%$, donde la producción de hilatura, tejeduría y acabados disminuyo un $8.3 \%$, el total de las ventas de la industria nacional creció un $0.8 \%$ en sector hilatura y tejeduría, $6.9 \%$ en el sector de acabados y el empleo solo creció un $1 \%$, a pesar de contar con el TLC y la 
Alianza del Pacifico (Lopera, 2015, diciembre).

Según Pérez (2016) el 71,5 \% del total de las unidades productivas del departamento de Antioquia se encuentran ubicadas en Medellín y son principalmente textileras, de sustancias y productos químicos. Así mismo, el PTP (2016) informa que, en el año 2015, Antioquia ocupó el segundo lugar con 34,2\% de las exportaciones en materiales textiles, después de Bogotá que tuvo un $54,7 \%$ de estas exportaciones.

El Sena, a través del Centro Textil y de Gestión Industrial de la Regional Antioquia y del Centro de Manufactura en Textil y Cuero de la Regional Distrito Capital, ofrece el programa de formación profesional integral en Tecnología Textil.Sin embargo, se ha venido observando una problemática asociada con el rendimiento académico de los aprendices.

Se estima que del orden del $20 \%$ al $30 \%$ de los aprendices del programa de formación en Tecnología Textil tienen que reforzar las pruebas de conocimiento de las competencias específicas, debido a las dificultades observadas en el manejo de los diferentes cálculos numéricos. Este hecho se ve agravado debido a que en los grupos de la modalidad de oferta cerrada el proceso de aprendizaje es más lento debido a que para los adultos que participan en ellos, muchos llevan años de no recibir formación titulada.

Por otra parte, según Garbanzo (2007) la didáctica empleada por los docentes es un determinante del proceso de aprendizaje: el uso de mapas conceptuales, las horas asignadas, la selección y organización de prácticas académicas repercuten en el rendimiento académico. Por esa razón se formuló la siguiente pregunta de investigación: ¿cómo están desarrollando, los docentes, la didáctica en su práctica educativa con las cohortes de tercero, quinto, séptimo y octavo trimestres en formación para la vigencia 2018, de la oferta cerrada del programa de Tecnología Textil, impartido en el Centro Textil y de Gestión Industrial del Sena en la ciudad de Medellín?

La Unesco (1998) resalta como misión de la educación superior, la de formar personas con altos niveles de cualificación, que a su vez sean ciudadanos responsables, capaces de atender a las necesidades de todos los aspectos de la actividad humana. La capacitación y las cualificaciones ofrecidas, que incluyen la capacitación profesional, deben estar a la altura de los tiempos modernos y combinar los conocimientos teóricos y prácticos de alto nivel, mediante cursos y programas que estén constantemente adaptados a las necesidades presentes y futuras de la sociedad.

En este contexto de la educación superior, la persona puede optar por una educación y formación técnica y profesional (EFTP) o por una educación universitaria. La Unesco-Unevoc (2010) define la Eftp como aquella parte de la educación que se ocupa de impartir conocimientos y destrezas o capacidades para el mundo del trabajo. Según el mismo organismo, a lo largo de la historia, los distintos países y sistemas educativos han utilizado términos diversos para referirse a varios elementos de lo que ahora se denomina la Eftp, incluyendo: formación de aprendices, educación o enseñanza profesional, educación técnica, educación técnico-profesional (ETP), formación ocupacional (FO), educación y formación profesional (EFP), educación profesional y de oficios, educación técnica y de carreras (ETC), formación o capacitación de la mano de obra, formación o capacitación para el puesto de trabajo, etc.

Según la Unesco-Unevoc (2010) el objetivo principal original de la Eftp era la 
preparación directa para el trabajo, lo cual sigue siendo válido para muchos países en vías de desarrollo. Sin embargo, las revoluciones tecnológicas y las innovaciones en ciencia y tecnología durante el siglo XX han dado importancia a nuevos ámbitos cognitivos y nuevas materias en todos los niveles educativos y formativos. Adicionalmente, el ascenso de la EFTP desde su categoría de educación primaria hasta la secundaria, y posteriormente hasta la terciaria, ha sido una evolución importante del siglo XX y marca la ruta para el siglo XXI.

El Servicio Nacional de Aprendizaje (Sena) nace como un establecimiento público de orden nacional en 1957 como iniciativa entre los trabajadores, los empresarios, la iglesia católica y la Organización Internacional del Trabajo, así mismo se encuentra adscrito al Ministerio de Trabajo y Seguridad Social de la República de Colombia; ofrece formación gratuita teóricopráctica para que las personas adquieran y mejoren destrezas, aptitudes, valores $y$ conocimientos técnicos y tecnológicos que les permitan desarrollar sus capacidades para actuar crítica y creativamente en la actividad productiva, contribuyendo así a su propio desarrollo personal, a la competitividad de la economía y al ejercicio pleno de la ciudadanía (Sena, 2017).

Según el Sena (s.f.) el Centro Textil y de Gestión Industrial, antes Ilamado Centro Nacional Textil, lleva más de 55 años de creado y en todos estos años ha logrado influenciar y responder a las necesidades del sector, al ser impulsor importante de la formación profesional integral, la asesoría, la investigación y los servicios tecnológicos. Este centro beneficia a la población de los Municipios del área metropolitana y a 17 municipios del norte de Antioquia. Desde su creación ha logrado elevar el nivel técnico y tecnológico de la industria textil y otros sectores económicos, con lo cual ha ayudado así a mejorar el nivel de vida del trabajador colombiano y apalancar la economía del país (Sena, s.f).

El programa de formación en Tecnología Textil se creó para brindar al sector productivo de las Tecnologías de Textil, confección diseño y moda, la posibilidad de incorporar personal con alta calidad laboral y profesional, atendiendo a las tendencias, cambios técnicos y las necesidades del sector empresarial y de los trabajadores.

Este programa es de nivel tecnológico, tiene oferta de formación abierta enfocada al público en general, así como cerrada, la cual está enfocada para grupos formados por trabajadores de las empresas del sector. Este programa es de carácter presencial, tiene una duración de ocho trimestres y puede participar cualquier colombiano que cumpla con el requisito mínimo de ser bachiller.

Por otra parte, según el Sena (2013) la Formación Profesional Integral como Proyecto Institucional tiene un papel estratégico al responder a las exigencias contemporáneas planteadas desde un proceso formativo estructurado por una dimensión humanista, en la que se evidencia la capacidad para la acción y la interacción del aprendiz consigo mismo, con el otro y con su entorno, desde una base sólida de valores, actitudes y emociones las cuales se integran para dar forma a las competencias básicas y por una dimensión cognitiva, y por otro lado por una dimensión cognitiva, en la que el aprendiz desarrolla de forma autónoma el conocimiento con el fin de ser aplicado en la resolución de problemas, con lo cual se evidencia el desarrollo de competencias de carácter específicas al enmarcarse en una línea tecnológica propia de cada programa de formación. El éxito en adquirir las competencias específicas 
depende en gran medida de la apropiación o aplicación de los conceptos adquiridos en las competencias básicas.

Para el desarrollo de competencias específicas antes descritas, se requiere la realización de cálculos numéricos utilizados para cálculos de producción para hilandería, tejido plano, tejido punto y no tejido; cálculo de la capacidad instalada, control de presupuesto, manejo de la información, costos de producción, estadística, conceptos matemáticos básicos, cálculos de promedio, desviaciones, coeficientes de variación, interpretación de gráficos, comparación con estadísticas nacionales e internacionales, cadenas cinemáticas en hilanderías, sistema y trenes de estiraje, sistemas de titulación y numeración de materiales textiles, porcentajes de mezcla de fibras, eficiencia de las máquinas, desarrollo de formulaciones químicas, balanceo de ecuaciones químicas, unidades de concentración de las soluciones acuosas, cálculos y formulación de los procesos de tintorería, entre otros; y es en la realización de estos cálculos donde los aprendices tienen las principales dificultades.

Estas dificultades se evidencian en el bajo resultado obtenido por la persona al realizar una prueba de conocimiento propuesta por el docente con el fin de evaluar el grado de apropiación de un tema en particular. Este bajo resultado muchas veces conduce a la no aprobación de la prueba, lo que implica que el aprendiz debe reforzarla.

Dentro de los determinantes institucionales y académicos que impactan directamente el rendimiento académico de los estudiantes, se incluyen aquellos aspectos propios de la misma institución de educación superior, de su administración, aspectos curriculares, de las propias asignaturas, y de la didáctica utilizada por los docentes, entre otros.

Durante mucho tiempo se tuvo la creencia que cualquier persona con nivel escolar y académico medio podía educar a las personas adultas, sin la necesidad de tener un conocimiento profesional didáctico específico acerca de los destinatarios de esa educación (Rumbo, 2016).

Sin embargo, Pérez (2009), afirma que el educador de adultos es un andragogo cuyo trabajo es ser un orientador, un facilitador más que un maestro en el sentido tradicional. Díaz Barriga y Hernández (como se citó en Caraballo, 2007) consideran que el docente se convierte en un organizador y mediador en el encuentro del aprendiz con el conocimiento.

Las necesidades cambiantes y complejas de la sociedad actual, así como los retos tecnológicos, han convertido la educación de adultos en un elemento indispensable del desarrollo personal y social, la cual ya no se ve como un correctivo educativo (Pérez, 2009).

Según la Unesco (1998), la educación de adultos comprende la educación formal y la permanente, la educación no formal y toda la gama de oportunidades de educación informal y ocasional que existen en una sociedad educativa multicultural, en la que se reconocen tanto los enfoques teóricos como los que se basan en la práctica (Pérez, 2009).

Las Instituciones de Educación Superior que trabajan con adultos, deben garantizar la coherencia y la pertinencia de sus programas de tal forma que busquen el desarrollo del país y no solamente responder a las expectativas de los adultos que buscan sus programas. 
Según Caraballo (como se citó en Bernal, 2017) con la andragogía la persona adulta realiza un proceso de educación integral en el cual la persona consciente no se limita a la adquisición de conocimientos, sino que mejora sus habilidades y destrezas de forma constante sobre un aspecto en particular, sino que se genera un crecimiento personal, profesional, social y de familia, para ser capaz de desenvolverse en una comunidad de la mejor forma posible.

Para este mismo autor, la andragogía estudia la realidad de la persona adulta y le define los lineamientos adecuados para dirigir su proceso de aprendizaje. Así mismo, Caraballo (2007) considera la andragogía como una disciplina cuyo fin es lograr aprendizajes significativos en los participantes adultos, al igual que promover la adquisición y la transferencia de conocimientos al contexto donde éste se desenvuelve.

El docente, como facilitador se encarga de perfeccionar las estrategias de enseñanza y aprendizaje como lo expone Caraballo (como se citó en Caraballo, 2007), con el fin de promover en los adultos la adquisición y retención de los conocimientos relevantes.

En este mismo sentido Torres et al., (2000) aduce que el facilitador ayuda a los participantes $\mathrm{o}$ estudiantes adultos a descubrirse como seres libres y responsables, colabora con ellos en la búsqueda del sentido y valor de su propia existencia.

El docente en la andragogía para Adam (como se citó en Caraballo, 2007) es el encargado de orientar, ayudar, cooperar con el estudiante en el logro de sus metas de aprendizaje, promover la reflexión, imaginación, creatividad, y mantener una relación horizontal, proactiva, recíproca, compartida, de responsabilidades, de negociación y de compromiso hacia logros y resultados exitosos.

Según Adam (como se citó en Torres et al., 2000) en el contexto de la andragogía, la relación entre el facilitador y el participante se encuentra enmarcada dentro del principio de horizontalidad, el cual establece características cualitativas y cuantitativas entre unos y otros. Características cualitativas ya que el participante y el facilitador son personas adultas que poseen experiencia; y características cuantitativas relacionadas con la disminución o merma que se presenta en la constitución física del adulto.

Según Educrea (s.f.) la didáctica adecuada para una persona que logra su adultez debe tomar en cuenta su correspondiente estado de madurez. El interrogante tradicional de cómo aprenden las personas adultas da paso a otro dilema y es: por qué, cómo y en qué condiciones se forman (Rumbo, 2016). La forma como aprenden los adultos, y en consecuencia la didáctica a utilizar, está en correspondencia con sus características.

La forma como el docente organiza los procesos y desarrolla la mediación tiene que ver con ladidáctica. La didáctica es la disciplina de la educación que se ocupa de organizar y administrar el proceso de enseñanza que un docente lleva a cabo para propiciar los aprendizajes de los estudiantes (Lucarelli, 2000 como se citó en Franco, 2013). Para efectos de esta investigación se consideró que la didáctica abarca seis sinergias: comunicación, planificación, facilitación, orientación, motivación y evaluación:

- Por comunicación se entiende aquellos aspectos del instructor que le permiten relacionarse con sus aprendices de forma adecuada. 
- La planificación se entiende como el proceso por medio del cual se establecen una serie de pasos y actividades encaminadas a lograr los diferentes objetivos de cada una de las asignaturas. La formulación de los objetivos de aprendizaje para los adultos debe ser un trabajo solidario entre el docente y el estudiante en los que se consideren sus necesidades, las de la institución educativa, las del docente y las de la sociedad (Pérez, 2009).

- Por facilitación se entiende como aquellas actividades realizadas por el instructor que permiten crear condiciones para el desarrollo adecuado y satisfactorio de los procesos de aprendizaje de los aprendices. Según Pérez (2009), un trabajo andragógico debe alejarse y diferenciarse de las lecciones impartidas de forma tradicional, de la lectura y discusión de textos y de la simple transmisión de contenidos. En este sentido Knowles (como se citó en Fasce, 2006), considera que se puede dar una instrucción a las personas adultas orientada hacia la tarea y no a la memorización del contenido.

- Por orientación se entiende la ayuda que presta el instructor a los aprendices para que sean capaces de resolver los problemas, situaciones o inquietudes de orden académico que se les presenten. Pérez (2009), considera que el adulto inicia un proceso de formación para enriquecer su cultura, su vida social, dinamizar su experiencia, contribuir a su bienestar y al de la sociedad a la que pertenece. Para García (1994), el tener un conocimiento adecuado de las dificultades, modelos, situaciones, estilos y motivos del aprendizaje adulto, facilitará la aplicación de las técnicas metodológicas adecuadas para facilitar el aprendizaje, así existan varias dificultades, el hecho de la amplia experiencia de los alumnos adultos posibilitará en mayor medida un aprendizaje significativo.

- La motivación es el proceso por medio del cual el instructor activa, dirige y mantiene la acción de aprendizaje en los aprendices. En investigaciones recopiladas por Dunn y Dunn (como se citó en García, 1994), se sugiere que los alumnos en su proceso de aprendizaje estaban afectados por la emotividad, lo que incluye la motivación, la persistencia, la responsabilidad y la necesidad de estructuración $\mathrm{o}$ de flexibilidad y las necesidades físicas. Por ejemplo, en un estudio realizado por Pérez (como se citó en Garbanzo, 2007) con estudiantes universitarios se destaca que la falta de motivación de los alumnos se refleja en aspectos como ausencia a clases, bajos resultados académicos, incremento de la repitencia $y$ en el abandono de sus estudios. Por otra parte, Pérez (2009) sostiene que otros factores que influyen en la motivación de las personas adultas en participar en procesos formativos, incluyen la oportunidad de intercambiar ideas con el grupo, cambiar de rutina, utilizar su tiempo libre, desarrollar su personalidad y aumentar sus ingresos. Entre los 18 y 40 años son mayores las motivaciones profesionales porque se considera que si se quiere disfrutar de la vida, debe prepararse en esta etapa (Torres et al., 2000).

- La evaluación abarca las estrategias empleadas por los instructores para recolectar información acerca del proceso de aprendizaje de los 
estudiantes a su cargo. La forma como se evalúa el aprendizaje también es uno de los factores que puede afectar el rendimiento.

Según Marín (como se citó en Pérez, 2009) al participar los adultos en procesos educativos no andragógicos es posible que los métodos de trabajo, los contenidos y los modos de evaluación no les complazcan no sean lo que necesitan o lo que desean, ni los puedan aplicar a la solución de sus problemas inmediatos. Es por esta razón que la didáctica a emplear con el adulto no puede ser escolarizada, sino que tiene que ser adaptada a sus actividades, tiempos y ocupaciones.

\section{MÉTODO}

Se llevó a cabo una investigación descriptiva, con el fin de caracterizar la didáctica utilizada por el docente con los aprendices del programa de Tecnología Textil. Se trabajó con un diseño de campo, transeccional, contemporáneo y univariable (Hurtado, 2010). Las unidades de estudio fueron los docenes del Programa de Tecnología textil, y los informantes fueron sus estudiantes. Se trabajó con los estudiantes que pertenecían a los cuatro grupos de Tecnología Textil en formación en el Centro Textil y de Gestión Industrial del Sena Medellín, a fínales del año 2018. Estos aprendices se encontraban cursando tercero, quinto, séptimo y octavo trimestre.El número total de unidades de estudio que participaron fue de 81 . Tabla 1 recoge la información demográfica de esos aprendices.

Tabla 1. Información demográfica de las unidades de estudio

\begin{tabular}{lrrrrrr}
\hline $\begin{array}{c}\text { Grupo } \\
\text { (Ficha) }\end{array}$ & Trimestre & $\begin{array}{c}\text { Cantidad } \\
\text { aprendices }\end{array}$ & $\begin{array}{c}\text { Cantidad } \\
\text { hombres }\end{array}$ & $\begin{array}{c}\text { Cantidad } \\
\text { mujeres }\end{array}$ & $\begin{array}{c}\text { Edad menor } \\
25 \text { años }\end{array}$ & $\begin{array}{c}\text { Edad mayor } \\
25 \text { años }\end{array}$ \\
\hline 1 & 8 & 16 & 10 & 6 & 6 & 10 \\
2 & 7 & 18 & 16 & 2 & 0 & 18 \\
3 & 3 & 20 & 9 & 11 & 3 & 17 \\
4 & 5 & 27 & 26 & 1 & 2 & 25 \\
\hline
\end{tabular}

Para la recolección de datos se utilizó un cuestionario que planteaba preguntas acerca de la didáctica manejada por los instructores, el cual constaba de 44 preguntas dicotómicas, de selecciones múltiples y mixtas, las cuales se agruparon en seis sinergias: comunicación, planificación, facilitación, orientación, motivación y evaluación. La confiabilidad total del instrumento fue de 0,80. El cuestionario arrojó un puntaje bruto total de 60 puntos. Para facilitar la interpretación, se transformó a una escala de 0 a 50 puntos. Para el caso de cada sinergia, su puntaje fue transformado también a la misma escala de 50 puntos, a fin de que las puntuaciones fuesen comparables. Para interpretar los puntajes obtenidos se utilizó la tabla 2 . 
Tabla 2. Intervalos y categorías del uso didáctico de las Tac

\begin{tabular}{ll}
\hline Intervalo & \multicolumn{1}{c}{ Categoría } \\
\hline $0-9,9$ & Muy deficiente \\
$10-19,9$ & Deficiente \\
$20-29,9$ & Regular \\
$30-39,9$ & Buena \\
$40-50$ & Muy buena \\
\hline
\end{tabular}

Para procesar los datos se empleó un análisis cuantitativo con técnicas estadísticas descriptivas, en las cuales se usaron medianas, y gráficos de caja y bigote.

\section{RESULTADOS Y DISCUSIÓN}

Se realizó un análisis estadístico de los datos obtenidos con el Cuestionario de didáctica aplicado a los 81 aprendices que hacían parte de los cuatro grupos en formación, a fin de obtener información acerca de lo que hace, durante el proceso didáctico, cada uno de los profesores de las tres asignaturas con alto porcentaje de contenido matemático (Proceso de tintorería, Proceso de hilandería y Control de calidad). La puntuación máxima transformada del cuestionario era de 50 puntos. La tabla 3, recoge los resultados para los grupos de octavo trimestre, séptimo trimestre, tercer trimestre y quinto trimestre de cada asignatura. 
Tabla 3. Resultado del análisis estadístico de la didáctica por grupo y asignatura

\begin{tabular}{|c|c|c|c|c|}
\hline Didáctica & Tintorería 8 & Tintorería 7 & Tintorería 3 & Tintorería 5 \\
\hline Mediana & 43,7500 & 43,3333 & 44,5833 & 46,6667 \\
\hline Mínimo & 34,17 & 25,83 & 39,17 & 39,17 \\
\hline Máximo & 48,33 & 48,33 & 48,33 & 50,00 \\
\hline \multicolumn{5}{|l|}{ Percentiles } \\
\hline 25 & 39,1667 & 40,2075 & 41,8750 & 44,1667 \\
\hline 50 & 43,7500 & 43,3333 & 44,5833 & 46,6667 \\
\hline 75 & 47,0833 & 45,4167 & 46,6667 & 47,5000 \\
\hline Didáctica & Hilandería 8 & Hilandería 7 & Hilandería 3 & Hilandería 5 \\
\hline Mediana & 45,8333 & 44,5833 & 45,8333 & 47,5000 \\
\hline Mínimo & 31,67 & 30,00 & 41,67 & 41,67 \\
\hline Máximo & 48,33 & 48,33 & 49,17 & 49,17 \\
\hline \multicolumn{5}{|l|}{ Percentiles } \\
\hline 25 & 43,5417 & 41,6667 & 44,1667 & 46,0417 \\
\hline 50 & 45,8333 & 44,5833 & 45,8333 & 47,5000 \\
\hline 75 & 47,2917 & 47,7083 & 47,5000 & 48,3333 \\
\hline Didáctica & Calidad 8 & Calidad 7 & Calidad 3 & Calidad 5 \\
\hline Mediana & 45,0000 & 43,7500 & 45,4167 & 45,8333 \\
\hline Mínimo & 36,67 & 37,50 & 43,33 & 42,50 \\
\hline Máximo & 47,50 & 46,67 & 46,67 & 47,50 \\
\hline \multicolumn{5}{|l|}{ Percentiles } \\
\hline 25 & 42,7083 & 43,1250 & 44,3750 & 45,0000 \\
\hline 50 & 45,0000 & 43,7500 & 45,4167 & 45,8333 \\
\hline 75 & 46,4583 & 45,8333 & 45,8333 & 46,6667 \\
\hline
\end{tabular}

El análisis estadístico tuvo en cuenta la mediana de cada asignatura en cada uno de los grupos. El resultado de este Cuadro se puede observar en el gráfico 1. 


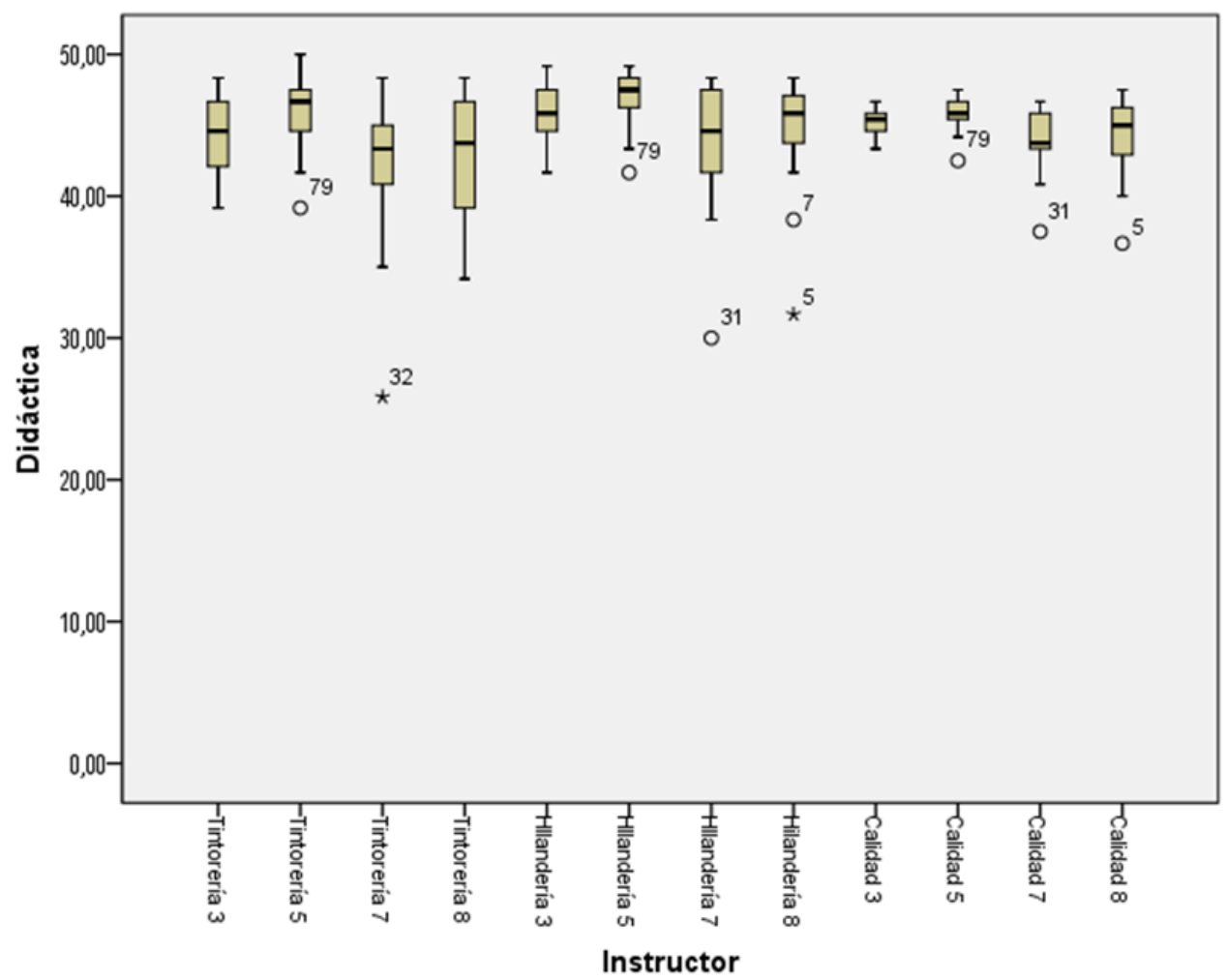

Gráfico 1. Medianas de didáctica en las tres asignaturas

Como se puede observar en el gráfico 1 , la didáctica de todos los instructores está ubicada dentro del rango de buena y muy buena, salvo algunos casos atípicos que se encuentran muy por debajo de la valoración del resto de los grupos. Todas las medianas están ubicadas en el rango muy bueno, con valores que van desde 43,33 hasta 47,5 en una escala de 50 puntos. Los mínimos están ubicados dentro del rango bueno con valores que van desde 30 hasta 42,5 puntos sobe 50 . Los valores máximos se ubican todos en el rango muy bueno, con valores que van desde 46,67 hasta 50.

Hay una pequeña variabilidad en el comportamiento de la didáctica de cada uno de los instructores para cada uno de los grupos. Para el caso del instructor de Proceso de tintorería, la mediana mínima fue 43,33 con el grupo de séptimo trimestre, la máxima fue de 46,67 con el grupo de quinto trimestre y su promedio estuvo en 44,58 .

La mediana mínima del instructor de Proceso de hilandería fue 44,58 con el grupo de séptimo trimestre, la máxima fue de 47,5 con el grupo de quinto trimestre y su promedio estuvo en 45,93.

Finalmente, el instructor de Control de calidad tuvo una mediana mínima de 43,75 con el grupo de séptimo trimestre, la máxima fue de 45,83 obtenida con el grupo de quinto trimestre y su promedio fue de 45,00 . Cabe destacar que al tener en cuenta el comportamiento de la mediana, los tres instructores tuvieron su valor mínimo con el grupo de séptimo trimestre y su valor máximo con el de quinto trimestre.

Al tener en cuenta los casos atípicos, se observó que el aprendiz identificado con el caso 79 , que pertenece al grupo de quinto 
trimestre, valoró de forma inferior a los tres instructores con respecto al resto de su grupo. Otro caso atípico a considerar fue el caso 5, que pertenece al grupo de octavo trimestre, y valoró de forma inferior que el resto de su grupo, al instructor de Control de calidad $y$ al instructor de Proceso de hilandería.El otro caso atípico fue el del caso 31, que pertenece al grupo de séptimo trimestre, que calificó por debajo de su grupo al instructor de Control de calidad, y muy por debajo al instructor de Proceso de hilandería.

\section{Discusión}

Se estudió la didáctica docente de los instructores de las tres asignaturas. Los resultados arrojaron que todas las medianas estaban ubicadas en el rango muy bueno, con valores que iban desde 43,33 hasta 47,5 sobre un máximo de 50 . Se observó que la mediana de los instructores tuvo su valor mínimo con el grupo de séptimo trimestre, con un valor de 43,33 para el instructor del proceso de tintorería, 44,58 para el instructor del proceso de hilandería y 43,75 para el instructor de control de calidad. Así mismo se observó que la mediana de los instructores tuvo su valor máximo con el grupo de quinto trimestre, con un valor de 46,67 para el instructor del proceso de tintorería, 47,5 para el instructor del proceso de hilandería y 45,75 para el instructor de control de calidad.

La facilitación incluyó indicios tales como técnicas de trabajo en grupo, técnicas orientadas al contenido, técnica de mapas conceptuales, técnicas dialógicas, técnica de aprendizaje basado en proyectos, técnica de resolución de problemas, así como complementar contenidos con actividades extracurriculares. Estos resultados están acordes con investigaciones realizadas por Roman (2015) las cuales arrojaron que se obtenían mejores resultados en las clases en las que la participación de los estudiantes era activa y se les ofrecía un repertorio variado de actividades que potenciaban el trabajo colaborativo entre ellos. Para el Sena, su finalidad principal es la formación profesional integral, donde una de las condiciones y exigencias básicas es el trabajo colaborativo. Todos los instructores deben implementarlo en sus asignaturas, sean estas de tipo teórico o teórico-práctico y es uno de los aspectos que deben evaluar en sus aprendices.

Otros indicios dentro de la sinergia de facilitación fueron la asignación de trabajos de investigación, el tener en cuenta el tiempo flexible para la realización de los trabajos, responder preguntas, realizar tareas de nivelación o reforzamiento, aclaración de dudas, así como asignación de trabajo para la casa. Para Roman (2015) estas son características de los docentes cuyos estudiantes avanzan y aprenden, y destaca la importancia de asignar trabajos y tareas dentro del aula y para la casa, así como de retroalimentar de manera oportuna a cada estudiante en cuanto a trabajos y tareas. Cabe anotar que la asignación de trabajos y tareas para la casa no es muy alta en el Sena dentro de la formación en Tecnología Textil, debido a que la mayoría de las actividades se realizan dentro del aula. Para el caso de las asignaturas que hacen parte de esta investigación, las labores principales que quedan para desarrollar en la casa son la finalización de los informes de prácticas y el repaso de los temas vistos.

Los indicios de la sinergia orientación, fueron el apoyo a los aprendices que tenían problemas, ofrecer sugerencias de estudio, ofrecer orientaciones para estudio independiente, así como dedicación de tiempo por parte del docente para atender 
de manera personal a los aprendices. Todos estos aspectos coinciden con la afirmación de Román (2015), con respecto a que un docente preocupado por conocer $y$ comprender los niveles de avances $y$ dificultades de sus alumnos dentro del proceso de aprendizaje puede apoyarlos de forma acorde y oportuna, de manera que avancen y logren apropiarse de los diferentes saberes y aprendizajes implicados. Así mismo, concuerda con el punto de vista de Latiesa (1992 como se citó en Vargas y Montero, 2016), cuando afirma que el rendimiento académico mejora cuando el profesorado es accesible, muestra interés y entusiasmo por la enseñanza, y organiza los tiempos, las formas de atención, las explicaciones y las aclaraciones. A este respecto, es muy habitual en el Sena el desarrollo por parte de los instructores de monitorias de facilitación extracurricular para sus aprendices, con el fin de responder las dudas y preguntas de ellos. Estas monitorias permiten mantener una comunicación fluida con los aprendices, adicional a los medios de comunicación habituales y tecnológicos disponibles en estos momentos.

Al evidenciarse que la dificultad principal para los aprendices estuvo en el uso de las matemáticas, es importante tener en cuenta otros aspectos dentro de la didáctica, en especial lo relacionadocon las características de los adultos. Hace parte pues de la labor del instructor el generar las estrategias didácticas adecuadas que les permitan a los aprendices adultos a su cargo, reducir al mínimo posible esa brecha existente entre lo abstracto y teórico de las matemáticas y su aplicabilidad en la vida laboral diaria. Esto lo confirman Merriam y Caffarella (1999 como se citó en Diez-Palomar, 2009) cuando afirman que los maestros de personas adultas deben usar la experiencia de sus estudiantes como un recurso para provocar aprendizaje y dotar de sentido a los conceptos que se están enseñando. Esta creación de sentido significa que se entiende lo que se está aprendiendo (Diez-Palomar, 2004 como se citó en Diez-Palomar, 2009).

\section{CONCLUSIONES}

La valoración de la didáctica utilizada por los instructores de las tres asignaturas consideradas, se ubicó dentro de las categorías buena y muy buena, y el comportamiento de los docentes fue bastante homogéneo en este evento. Las sinergias que más trabajaron con fueron facilitación yorientación. En el proceso de facilitación los docentes tienden a utilizar técnicas de trabajo en grupo, técnicas orientadas al contenido, mapas conceptuales, técnicas dialógicas, aprendizaje basado en proyectos, y resolución de problemas. En cuanto a la sinergia orientación, los docentes tienden a mostrar apoyo a los aprendices que con problemas, ofrecer sugerencias de estudio, ofrecer orientaciones para estudio independiente, y dedican de tiempo para atender de manera personal a los aprendices.

\section{REFERENCIAS}

Bernal, J. (2017). Evolución conceptual e importancia de la andragogía para la optimización del alcance de los programas y proyectos académicos universitarios de desarrollo rural. Revista Electrónica Educare, 21(1), 1-16. Recuperado de https://dialnet.unirioja.es/servlet/articul o?codigo $=5763966$

Caraballo, R. (2007). La Andragogía en la Educación Superior. Investigación y Postgrado, 22(2). Recuperado de http://revistas.upel.edu.ve/index.php/re vinpost/article/view/678 
Conde, J. (2009). La Innovación como determinante de la competitividad en la industria textil - confección: el caso de Corea y Colombia (tesis de pregrado). Pontificia Universidad Javeriana, Bogotá, Colombia. Recuperado de http://javeriana.edu.co/biblos/tesis/eco nomia/tesis56.pdf

Diez-Palomar, J. (2009). La enseñanza de las matemáticas a personas adultas desde un enfoque didáctico basado en el aprendizaje dialógico. Enseñanza de las ciencias, 27(3), 369-380. Recuperado de https://www.raco.cat/index.php/Ensena nza/article/viewFile/142070/332852

Educrea. (s.f). Estilos y Metodologías de Aprendizaje. Santiago: Educrea. Recuperado de

https://educrea.cl/estilos-ymetodologias-de-aprendizaje/

Fasce, E. (2006). Andragogía. Revista de Educación en Ciencias de la Salud, 3(2), 69-70. Recuperado de http://www2.udec.cl/ofem/recs/anterio res/vol322006/esq32.pdf

Franco, J. (2013). La didáctica en el que hacer del docente, para facilitar el proceso de enseñanza en enfermería (tesis de especialización). Universidad Militar Nueva Granada, Bogotá, Colombia. Recuperado de https://repository.unimilitar.edu.co/bits tream/handle/10654/10462/FrancoBarr aganJennyEsmeralda2013.pdf?sequence $=1$

Garbanzo, G. (2007). Factores asociados al rendimiento académico en estudiantes universitarios, una reflexión desde la calidad de educación superior pública. Revista Educación, 31(1), 43-63. Recuperado de https://revistas.ucr.ac.cr/index.php/edu cacion/article/view/1252/1315

García, L. (1994). El aprendizaje a distancia de las personas adultas. En L. García, Educación a distancia hoy (pp. 109-176). Universidad Nacional de Educación a Distancia (Uned). Recuperado de https://www.researchgate.net/publicati
on/235464167_Educacion_a_distancia_ hoy

González, M. (23 de enero de 2018). Textil y confección son el 2,5\% del PIB de Antioquia. Portafolio. Recuperado de https://www.portafolio.co/negocios/por centaje-que-aportan-textil-y-confeccional-pib-de-antioquia-513556

Hurtado, J. (2010). Metodología de la investigación: Guía para una comprensión holística de la ciencia. Bogotá, Colombia: Quirón-Sypal

Jara, M. (2000). Estudio de Caracterización Ocupacional del Sector Fibras Textil Confección en Colombia, Prospectiva Tecnológica y Talento Humano. Recuperado de https://repositorio.sena.edu.co/bitstrea m/11404/4001/1/3975_estudio_de_car acterizacion_ocupacional_del\%20sector _de_fibras.pdf

Lopera, O. (2015, diciembre). Mesa sectorial industria textil, reunión Consejo General 2015 [Acta de la reunión]. Medellín, Colombia.

Ministerio de Educación Nacional de Colombia. (2015). Plan Nacional de Desarrollo 2014-2018. Recuperado de https://www.mineducacion.gov.co/1759 /articles-357047_recurso_1.pdf

Pérez, L. (2016). Bases del Plan de Desarrollo de Antioquia: "Pensando en Grande 2016-2019". Recuperado de http://www.asambleadeantioquia.gov.c o/2016/images/articles/planDesarrollo. pdf

Pérez, S. (Comp). (2009). Modelo Andragógico Fundamentos. México D.F, México: Universidad del Valle de México. Recuperado de https://my.laureate.net/faculty/docs/Fa culty\%20Documents/Andragogia.Funda mentos.pdf

Programa de Transformación Productiva. (2016). Informe de Textiles y Confección 2016. Recuperado de http://www.ptp.com.co 
Román, M. (2015). Investigación latinoamericana sobre enseñanza eficaz. Educación y ciudad. 19, 81-96. Recuperado de http://revistas.idep.edu.co/index.php/e ducacion-y-ciudad/article/view/121/110

Rumbo, B. (2016). Problemas y retos de la educación de las personas adultas. Revista Educar, 52(1), 93-106. Recuperado de http://educar.uab.cat/article/view/v52n1-rumbo/707-pdf-es

Sena. (2013). Proyecto Educativo Institucional Sena. Bogotá: Servicio Nacional de Aprendizaje, Dirección General. Recuperado de https://repositorio.sena.edu.co/bitstrea m/11404/3253/1/pei_sena.pdf

Sena. (2017). El Sena, quienes somos. Recuperado de http://www.sena.edu.co/esco/sena/Paginas/quienesSomos.aspx

Torres, M., Fermín, Y., Arroyo, C., Piñero, M. (2000). La Horizontalidad y la Participación en la Andragogía. Educere, Artículos 4(10), 25-33. Recuperado de http://www.saber.ula.ve/bitstream/han dle/123456789/19444/articulo4-10-

3.pdf?sequence $=1$

Unesco. (1998). Declaración mundial sobre la educación superior en el siglo XXI: visión y acción. Recuperado de http://www.unesco.org/education/educ prog/wche/declaration_spa.htm

Unesco-Unevoc. (2010). ¿Qué es la EFTP? Recuperado de https://unevoc.unesco.org/go.php?q=tr asfondo+Qu\%C3\%A9+es+la+EFTP\%3F+\& context=

Vargas, M., Montero, E. (2016). Factores que determinan el rendimiento académico en Matemáticas en el contexto de una universidad tecnológica: aplicación de un modelo de ecuaciones estructurales. Universitas Psychologica, 15(4).

Recuperado de https://www.google.com/url?sa=t\&rct=j $\& q=\& e s r c=s \&$ source $=$ web $\& c d=1 \& c a d=r j$ a\&uact $=8$ \&ved $=2$ ahUKEwjb1aXJsuTfAhV EiFkKHepKByYQFjAAegQICRAB\&url=http s\%3A\%2F\%2Frevistas.javeriana.edu.co\% 2Findex.php\%2FrevPsycho\%2Farticle\%2 Fview\%2F7502\%2F14266\&usg=AOvVaw Oid7dTwgrNmhSX9HrCfcnL 